\title{
4
}

\section{Vibrio cholerae Flagellar Synthesis and Virulence}

\author{
Anastasia R. Rugel ${ }^{1,2}$ and Karl E. Klose ${ }^{1}$ \\ ${ }^{1}$ South Texas Center for Emerging Infectious Diseases, Department of Biology, \\ University of Texas at San Antonio, San Antonio, Texas \\ ${ }^{2}$ Department of Microbiology and Immunology, \\ University of Texas Health Science Center at San Antonio, San Antonio, Texas \\ USA
}

\section{Introduction}

Vibrio cholerae is a Gram-negative bacterium with a single sheathed polar flagellum (Fig. 1.). $V$. cholerae causes the severe diarrheal disease cholera in humans when it colonizes the small intestine and expresses various virulence factors, including cholera toxin (CT) and toxin coregulated pilus (TCP). $V$. cholerae is also a natural inhabitant of the marine environment, where it forms biofilms on chitinous surfaces. Motility contributes to both aspects of the $V$. cholerae lifecycle. The flagellum facilitates chemotactic-directed movement toward the preferred colonization site within the intestine (Camilli and Mekalanos 1995; Butler and Camilli 2004), and also contributes to biofilm formation within the environment (Watnick and Kolter 1999). V. cholerae strains defective for motility are less virulent than motile strains (Guentzel and Berry 1975; Freter and O'Brien 1981; Richardson 1991). As flagellar synthesis, motility, and chemotaxis have become better understood in $V$. cholerae, it has also become clear that motility is intimately integrated into all aspects of the lifestyle of this bacterium.

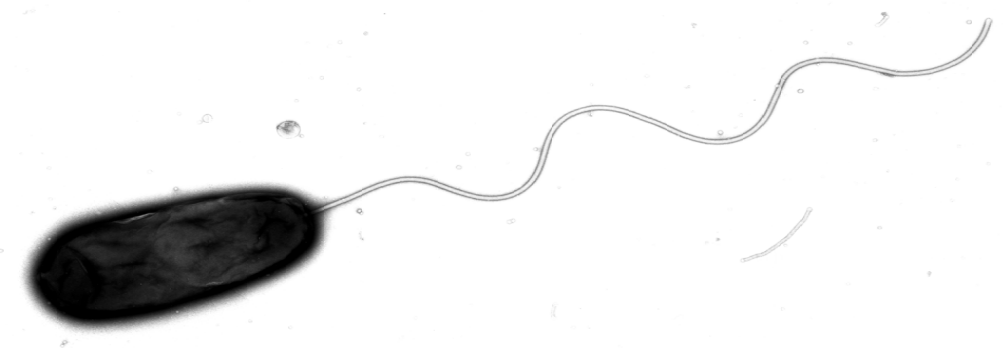

Fig. 1. Vibrio cholerae

\section{Structure}

The flagellum is a motor-driven organelle present in many bacteria. Different flagellar placement and quantity are seen in different bacteria. Monotrichous bacteria have a single 
polar flagellum (e.g. V. cholerae), lofotrichous bacteria have multiple flagella at a single pole (e.g. Helicobacter pylori), amphitrichous bacteria have flagella at two poles (e.g. Campylobacter jejuni), and peritrichous bacteria have multiple flagella emanating from the cell in all directions (e.g. Escherichia coli).

The base of the bacterial flagellum is composed of a secretion system related to the Type III secretion system, which facilitates export of flagellar components from the cytoplasm to the periplasm and the exterior of the cell. The basic components of the flagellum are the basal body, which extends from the cytoplasmic membrane through the periplasm and into the outer membrane $(\mathrm{OM})$, connected to the flexible hook (composed of FliE) found exterior to the cell, which in turn is connected to the flagellar filament (Kojima and Blair 2004; Terashima, Kojima et al. 2008). The motor components that drive flagellar rotation are found in the cytoplasmic membrane, and the switch components (FliG, FliM, FliN) that interact with the chemotaxis signaling system and the motor (Francis, Sosinsky et al. 1994) extend into the cytoplasm from the basal body (Fig. 2.).

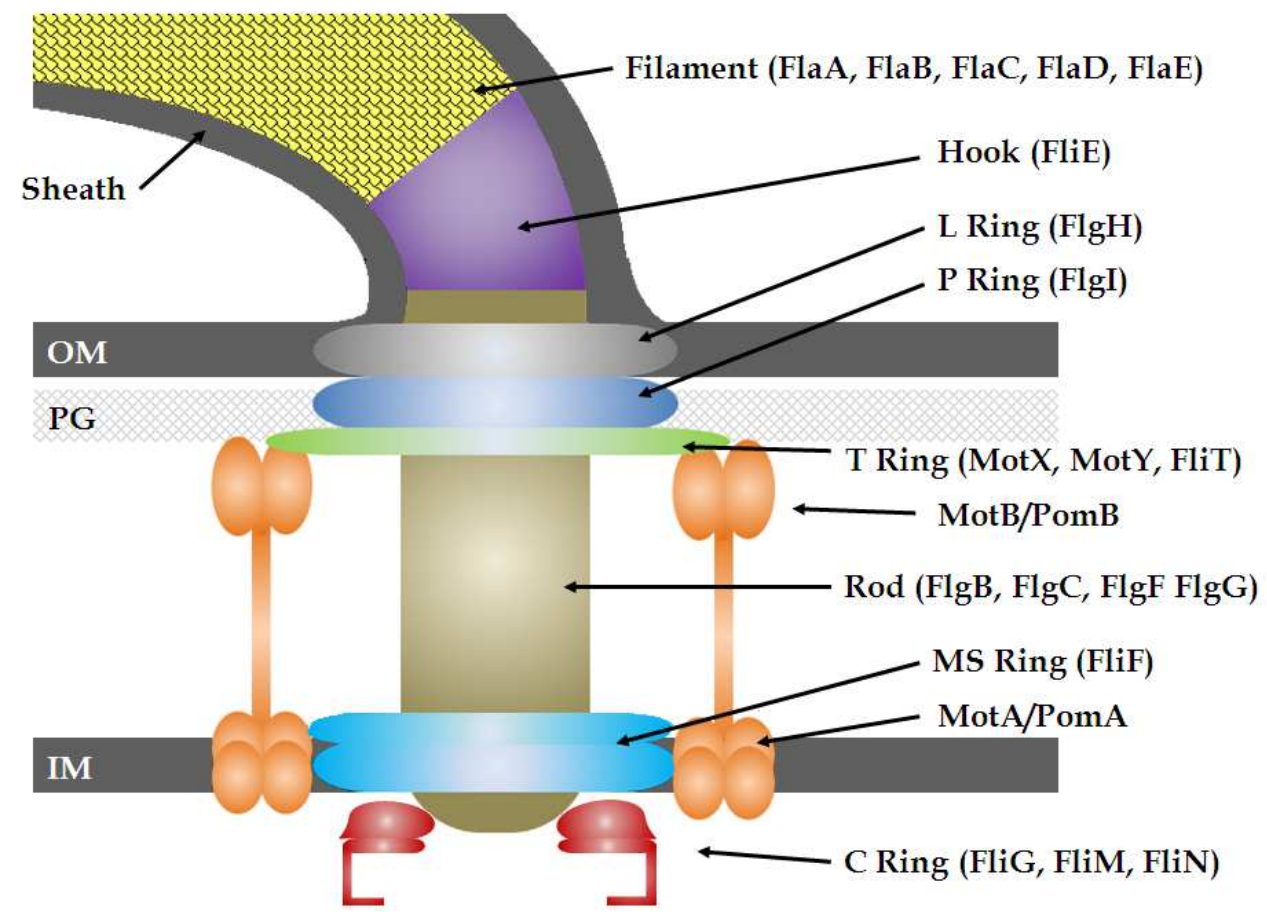

Fig. 2. Flagellar Motor Complex

Unlike most other bacterial flagella, the $V$. cholerae flagellum has a sheath composed of OM that coats the entire filament (Allen and Baumann 1971; Sjoblad, Emala et al. 1983; Fuerst and Perry 1988). Sheathed flagella are found in Vibrio spp. and a few other Gram-negative bacteria (e.g. H. pylori). It is hypothesized that the sheath acts as a protective covering that shields the antigenic flagellins from recognition by the host's immune response (Yoon and Mekalanos 2008). The mechanism whereby the OM is extended to cover the filament during 
$V$. cholerae flagellar synthesis rather than the filament protruding through the $\mathrm{OM}$ as in other bacterial flagella is not understood.

The bacterial flagellar filament is made up of thousands of flagellin subunits, with a cap protein (FliD) at the distal end (Ikeda, Asakura et al. 1985; Ikeda, Homma et al. 1987; Homma, DeRosier et al. 1990). The structure of the Salmonella typhimurium flagellin FliC has been solved by cryomicroscopy. FliC is composed of domains at its $\mathrm{N}$ - and C-termini that interact with each other: D0 (aa 1-45 and 456-495), D1 (aa 46-180 and 408-455), and D2 (aa 181-190 and 285-407). The D2 domains, along with the D3 domain (aa 191-284) form the antigenic variable region that are present on the filament surface, (Yonekura, MakiYonekura et al. 2003). Interaction of the D0 and D1 domains allows the flagellins to polymerize under the cap (FliD) protein into a hollow helical filament at the growing tip of the flagellum as they are being secreted.

In contrast to most other bacteria which have filaments composed of a single flagellin subunit, $V$. cholerae has a filament composed of 5 different flagellins, FlaABCDE. These flagellins share a high degree of homology, yet only FlaA is essential for flagellar synthesis; the other four flagellins are not required for the synthesis of the filament (Klose and Mekalanos 1998). Alignment of FlaA with the other four $V$. cholerae flagellins, as well as with S. typhimurium FliC, reveals that the D0 and D1 domains are well-conserved, whereas the variable regions D2 and D3 are more divergent. Interestingly, the $V$. cholerae flagellins have a much shorter region corresponding to D2 and D3 (129 aa shorter) when compared to $S$. typhimurium FliC. Because this antigenic portion of the flagellins extends out from the hollow filament core, it may be that the presence of the flagellar sheath over the $V$. cholerae filament restricts the size of the antigenic region protruding from the filament.

The basal body contains the rod structure (FlgB, FlgC, FlgF and FlgG) with L (FlgH), P (FlgI), and MS rings (FliF) localized to the OM, periplasm (peptidoglycan), and cytoplasmic membranes, respectively. In Vibrio spp. an additional $\mathrm{T}$ ring is located immediately below the $\mathrm{P}$ ring, which is composed of the Vibrio-specific components $\operatorname{Mot} X, \operatorname{Mot} Y$, and FlgT . The C-ring, which extends into the cytoplasm from the MS ring and is made up of FliG, FliM, and FliN, is difficult to preserve during microscopy and has not been visualized in its entirety in Vibrio spp.(Aizawa, Dean et al. 1985; Homma, Aizawa et al. 1987; Homma, Ohnishi et al. 1987; Homma, DeRosier et al. 1990; Homma, Kutsukake et al. 1990; Ueno, Oosawa et al. 1992; Francis, Sosinsky et al. 1994; Schoenhals and Macnab 1996; Terashima, Koike et al. 2010). The chemotaxis protein CheY relays information from the chemotaxis sensory system by binding to the $\mathrm{C}$ ring (FliM), causing the flagellum to switch rotation from counterclockwise to clockwise.

In S. typhimurium and E. coli MotA and MotB are membrane proteins that compose the motor that utilizes H+ motive force to drive flagellar rotation (Lloyd, Tang et al. 1996; Zhou, Lloyd et al. 1998; Zhou, Sharp et al. 1998; Braun, Poulson et al. 1999; Blair 2003). Vibrio spp. contain MotA and MotB homologues, alternately referred to as PomA and PomB (Dean, Macnab et al. 1984; Stader, Matsumura et al. 1986; Blair and Berg 1990; Stolz and Berg 1991; Asai, Kojima et al. 1997; Sato and Homma 2000; Sato and Homma 2000; Yorimitsu, Asai et al. 2000; Fukuoka, Yakushi et al. 2005), but they also contain Vibrio- specific motor proteins MotX and MotY, localized in the T ring (McCarter 1994; McCarter 1994; Okunishi, Kawagishi et al. 1996; Okabe, Yakushi et al. 2001; Okabe, Yakushi et al. 2002; Okabe, 
Yakushi et al. 2005; Koerdt, Paulick et al. 2009). The Vibrio MotA and MotB form a membrane complex that utilizes a $\mathrm{Na}+$ gradient (instead of $\mathrm{H}+$ gradient) to drive flagellar rotation. A Na+ gradient is required to allow MotA/MotB to associate with the flagellum (through MotX/MotY) and open the Na+ channel; flux of $\mathrm{Na}+$ through the channel provides the torque to generate flagellar rotation (McCarter 1994; McCarter 1994; Yorimitsu, Kojima et al. 2004; Terashima, Fukuoka et al. 2006).

Two additional proteins control flagellar number and placement in Vibrio spp. FlhG contains an ATPase motif and controls flagellar number; Vibrio cells without flh $G$ synthesize multiple polar flagella, instead of a single polar flagellum (Correa, Peng et al. 2005; Kusumoto, Kamisaka et al. 2006; Kusumoto, Shinohara et al. 2008). FlhF contains a GTP binding motif and localizes to the cell pole, thus dictating polar localization of the flagellum. Vibrio cells without $f h F$ are largely non-flagellated; however a few cells will synthesize a flagellum at a site away from the pole (Carpenter, Hanlon et al. 1992; Zanen, Antelmann et al. 2004; Salvetti, Ghelardi et al. 2007; Green, Kahramanoglou et al. 2009; Kusumoto, Nishioka et al. 2009). FlhG interacts with FlhF, and a current model suggests that FlhG interacts with FlhF to prevent additional FlhF deposition at the pole (Kusumoto, Shinohara et al. 2008). A V. alginolyticus strain lacking both FlhF and FlhG is mostly lacking flagella (Kojima, Nishioka et al. 2011), but a few cells possess multiple peritrichous flagella (similar to S. typhimurium). An unidentified suppressor mutation can lead to virtually all flhFG $V$. alginolyticus cells possessing peritrichous flagella and being able to swim; the identification of this suppressor mutation should lead to greater insights into control of polar flagellar synthesis in Vibrio spp.

Two additional outer membrane proteins, $\mathrm{FlgO}$ and $\mathrm{FlgP}$, contribute to flagellar stability. FlgP homologues are restricted to Vibrio, Helicobacter, and Campylobacter spp. V. cholerae FlgP is a lipoprotein that affects flagellar stability; $f l g P$ mutants synthesize fragile flagella and appear non-motile in motility agar, presumably due to breakage of flagella during swimming (Morris, Peng et al. 2008; Martinez, Dharmasena et al. 2009). FlgO homologues are only found in Vibrio spp. $V$. cholerae strains lacking flgO have a similar phenotype as flgP strains, namely they produce fragile flagella that break easily while swimming (Morris, Peng et al. 2008; Martinez, Dharmasena et al. 2009).

\section{Regulation}

Transcription of the $V$. cholerae flagellar genes is controlled by a four-tiered transcription hierarchy (Fig. 3.) (Prouty, Correa et al. 2001). The V. cholerae flagellar transcription hierarchy is similar to that which controls flagellar transcription in Pseudomonas aeruginosa, another bacterium with a single polar flagellum (Dasgupta, Wolfgang et al. 2003). The master regulator, FlrA, is a $\sigma^{54}$-dependent transcriptional activator. FlrA represents the sole Class I gene product, and it activates transcription of Class II flagellar genes (Klose and Mekalanos 1998). It is not clear whether environmental conditions regulate transcription of flrA, but $f l h G$ (which controls flagellar number) also negatively regulates flr $A$ transcription (Correa, Peng et al. 2005).

The $P$. aeruginosa FlrA homologue, FleQ, has been shown to bind to cyclic-di-GMP (cdGMP)(Hickman and Harwood 2008). Binding of cdGMP to FleQ prevents DNA binding, resulting in the absence of flagellar synthesis and de-repression in $P$. aeruginosa of genes involved in biofilm formation normally repressed by FleQ. Interestingly, cdGMP binds to 
FleQ lacking the N-terminus, indicating it binds to the transcriptional activation/DNA binding domain, which shares high homology (63\% identity) with $V$. cholerae FlrA. It is not yet known whether FlrA binds to and is modulated by cdGMP. P. aeruginosa FleQ also binds to FleN, the homologue of FlhG (Dasgupta and Ramphal 2001). FleN binding to FleQ does not inhibit DNA binding, but downregulates FleQ-dependent transcription, resulting in reduced (single) flagellar number. As mentioned above, FlhG has a negative effect on flrA transcription in $V$. cholerae, but it is not known whether it also binds to FlrA and negatively affects its activity.

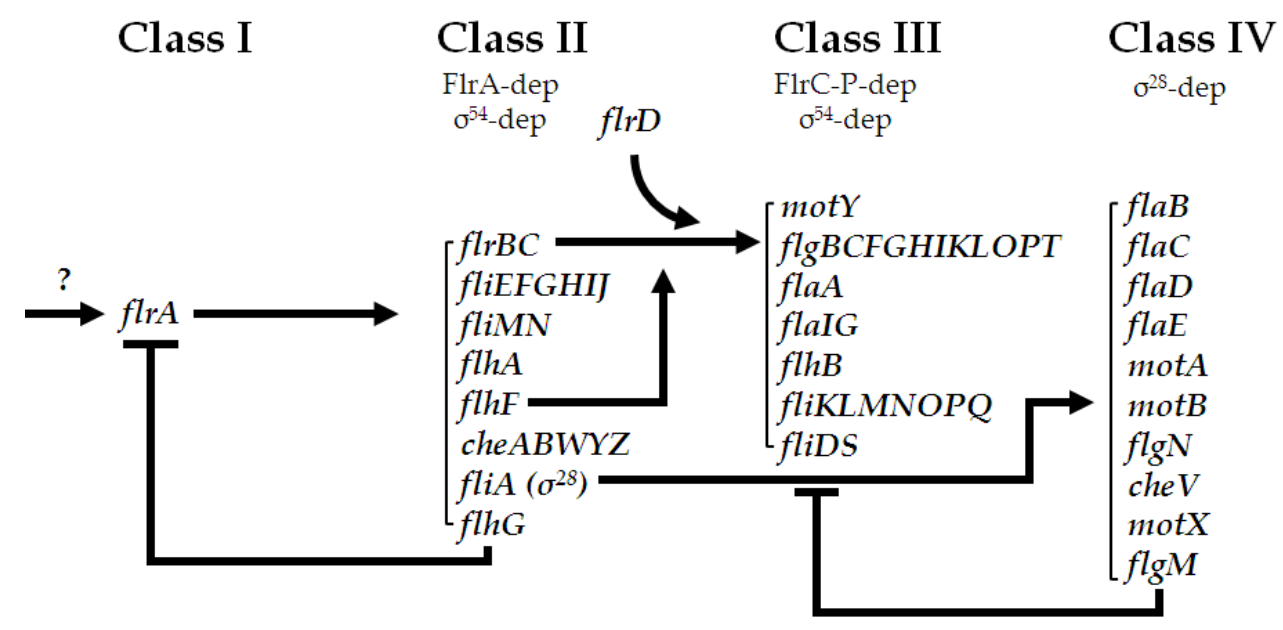

Fig. 3. Flagellar Transcription Regulatory Hierarchy

FlrA positively regulates Class II flagellar genes. Both FlrA and $\sigma^{54}$-containing RNA polymerase are required to activate transcription of the Class II flagellar genes (Klose and Mekalanos 1998; Klose, Novik et al. 1998; Prouty, Correa et al. 2001). The class II genes encode components of the MS ring-switch-export apparatus as well as chemotaxis and regulatory proteins. Two large flagellar operons (fliEFGHIJ and the flhA operon, which contains flhFG, mentioned above, as well as fliA $\left(\sigma^{28}\right)$ and a number of chemotaxis genes), and the regulatory genes $f l r B C$, are activated by FlrA. The Class II flagellar genes are predicted to encode an export apparatus-basal body intermediate; it seems likely that this structure is required to be assembled prior to progression to Class III gene expression, as is the case in Campylobacter jejuni and Helicobacter pylori, which have similar classes of flagellar genes (Hendrixson and DiRita 2003; Niehus, Gressmann et al. 2004).

The regulatory proteins FlrBC are a two-component system that controls Class III gene transcription (Prouty, Correa et al. 2001). FlrB undergoes autophosphorylation, and then activates FlrC activity by transferring a phosphate to the conserved aspartate-54 (D54) residue in the amino terminus of FlrC (FlrC-P) allowing it to activate the $\sigma^{54}$-dependent transcription of Class III genes (Correa, Lauriano et al. 2000; Correa and Klose 2005). The class III genes encode the rest of the components of the hook-basal body, as well as the flagellin FlaA and the OM proteins FlgOP. FlrC binds to enhancer sites downstream of the $0^{54-d e p e n d e n t ~ C l a s s ~ I I I ~ p r o m o t e r s ~(C o r r e a, ~ L a u r i a n o ~ e t ~ a l . ~ 2000 ; ~ C o r r e a ~ a n d ~ K l o s e ~ 2005) . ~}$ 
Most of the Class III gene products are only required in small amounts, but the FlaA flagellin is transcribed at very high levels. One mechanism for achieving these different levels of expression is the relative binding strength of the FlrC sites, which bind FlrC strongly at the flaA promoter, but only weakly at other Class III promoters, e.g. the flgK promoter (Correa and Klose 2005).

FlrC must be phosphorylated to activate $\sigma^{54}$-dependent transcription, so presumably FlrB only phosphorylates FlrC upon assembly (not function) of the Class II export apparatusbasal body intermediate; a similar event controls expression of $\sigma^{54}$-dependent Class III genes in C. jejuni (Joslin and Hendrixson 2009). Detection of an intermediate that is not secretion competent may explain why the genes encoding some of the components presumably required for secretion (e.g. fliOPQ) are Class III (i.e. activated by FlrC) rather than Class II genes. FlrB is a soluble protein and could thus directly interact with the apparatus intermediate in the cytoplasmic membrane and phosphorylate FlrC upon assembly. Deletion of $f l h F$ in $V$. cholerae specifically downregulates Class III gene expression (Correa, Peng et al. 2005), suggesting that FlhF regulates FlrC-dependent transcription in addition to regulating polar flagellar placement (as discussed above). An inner membrane protein, FlrD, is also a positive regulator of class III genes. Expression of FlrD is not regulated by the flagellar transcription hierarchy, but the protein possesses a HAMP domain, so it may interact with FlrB or FlrC to influence phosphorylation and Class III transcription (Moisi, Jenul et al. 2009)

The Class II gene fliA encodes $\sigma^{28}$, which is required for transcription of Class IV flagellar genes (Klose and Mekalanos 1998). Similar to the checkpoint in S. typhimurium (Karlinsey, Tanaka et al. 2000; Chevance and Hughes 2008), the $V$. cholerae anti-sigma factor FlgM prevents $\sigma^{28}$ transcriptional activity until it is secreted through a functional hook-basal body complex (Correa, Barker et al. 2004). The secretion of FlgM through the sheathed flagellum indicates that the sheath does not completely enclose the flagellum, at least at the tip. Secretion of FlgM frees $\sigma^{28}$ to interact with RNA polymerase and activate Class IV flagellar genes, which encode the other four flagellins, FlaBCDE, as well as motor components (MotABX) and chemotaxis proteins (Klose and Mekalanos 1998). V. cholerae lacking fliA are non-motile and synthesize a truncated flagellum. The lack of expression of the four additional Class IV ( $\sigma^{28}$-dependent) flagellins (FlaBCDE) in the fliA strain is likely not the reason for the truncated flagellum and lack of motility, since strains lacking flaBCDE are still motile and synthesize a full length flagellum, whereas a strain lacking the Class III FlaA flagellin is non-motile and aflagellate (Klose and Mekalanos 1998). Rather, the lack of expression of other Class IV genes (e.g. motor genes) likely contributes to the fliA phenotype. The contribution of the four Class IV flagellins to flagellar synthesis and motility is mysterious, considering that only the Class III FlaA flagellin is essential for flagellar synthesis, but perhaps the other flagellins impart subtle differences to the flagellum and thus swimming behavior that are not obvious under laboratory growth conditions.

\section{Motility and virulence}

$V$. cholerae virulence has been linked to motility. Spontaneous non-motile $V$. cholerae strains were characterized as less virulent than motile strains in several in vivo and in vitro rabbit models of cholera. Mutations that adversely affect flagellar synthesis and motility generally lead to decreased intestinal colonization in infant mice (Guentzel and Berry 1975; Montie, 
Doyle-Huntzinger et al. 1982; Carsiotis, Weinstein et al. 1984; Weinstein, Carsiotis et al. 1984; Schmitt, Darnell et al. 1994; Kennedy, Rosey et al. 1997; Watnick, Lauriano et al. 2001; Syed, Beyhan et al. 2009). Non-motile live attenuated $V$. cholerae vaccine strains exhibit reduced reactogenicity (disease symptoms) in human volunteers, when compared to motile isogenic strains. (Coster, Killeen et al. 1995; Kenner, Coster et al. 1995). Using a newly-developed infant rabbit model of cholera, Rui et al. demonstrated that flagellin expression (whether in motile or non-motile vaccine strains) causes reactogenicity in rabbits by inducing proinflammatory cytokines in the intestine (Rui, Ritchie et al. 2010).

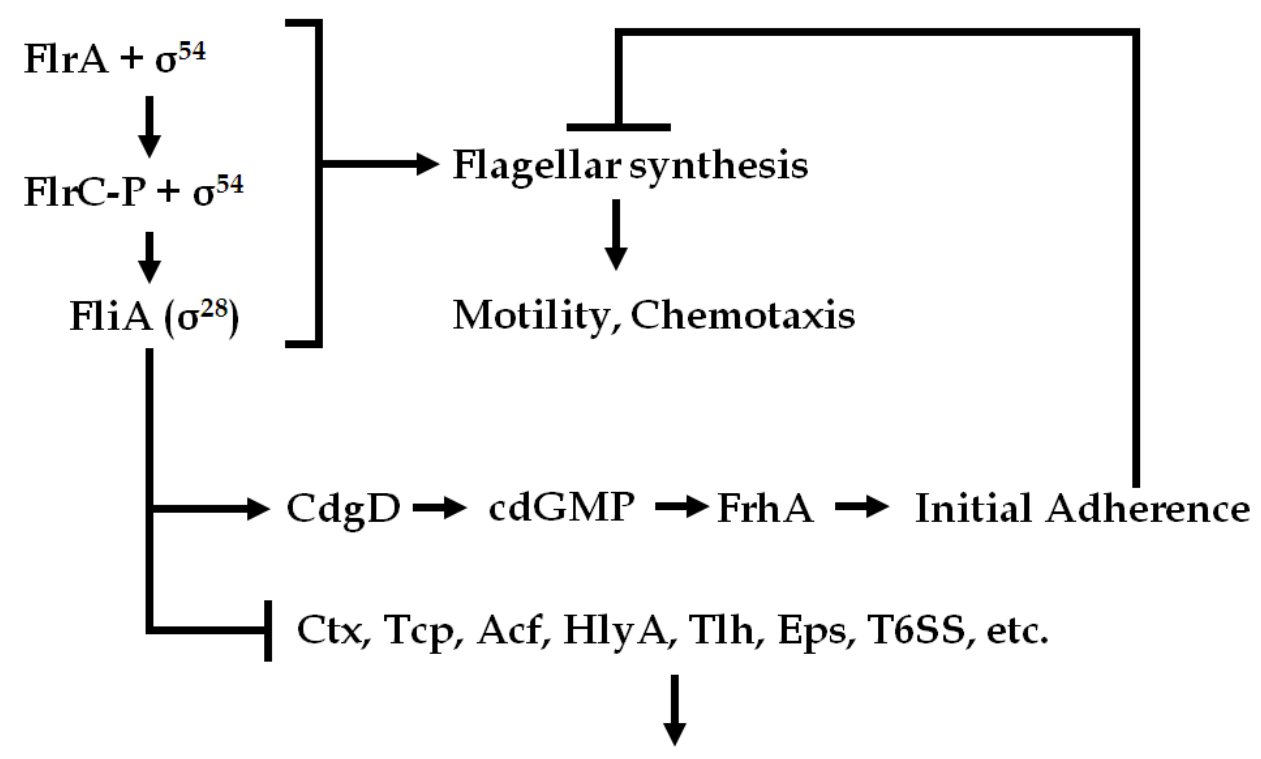

\section{Persistent colonization}

Fig. 4. Proposed Model of Flagellar-dependent Virulence Modulation

An inverse relationship between motility and virulence had been suggested by the observation that spontaneous hypermotile mutants express almost no CT or TCP, while spontaneous non-motile mutants express increased levels of CT and TCP (Gardel and Mekalanos 1996). Utilizing whole genome transcription profiling of $V$. cholerae strains with mutations in the key flagellar regulatory genes $(r p o N, f l r A, f l r C$, and $f l i A)$, it was observed that non-flagellated strains exhibit increased transcription of known (CT, TCP) and putative virulence factors (T6SS, hemolysins, etc)(Syed, Beyhan et al. 2009). The results suggest coordinate regulation by the flagellar regulatory hierarchy over a variety of virulence factors whose regulation was previously thought to be unlinked (Syed, Beyhan et al. 2009).

It had been known that non-motile $V$. cholerae mutants exhibited enhanced hemagglutinating activity and decreased hemolytic activity, but the identity of the respective factors was unknown (Gardel and Mekalanos 1996). The transcriptional profiling of the flagellar regulatory mutants identified the flagellar-regulated hemolysin as TLH, which is encoded adjacent to HlyA, the "El Tor" hemolysin (Syed, Beyhan et al. 2009). Also identified was the flagellar-regulated hemagglutinin, FrhA, which is a large cadherin- 
containing protein that enhances binding to epithelial cells in vitro and intestinal colonization in both infant and adult mice. The flagellar regulatory hierarchy positively regulates frh $A$ transcription and negatively regulates th transcription. Regulation of frh $A$ transcription by the flagellar hierarchy is mediated through an intermediate, CdgD, a cdGMP synthase. cdGMP is an important signaling molecule that modulates complex behaviors in bacteria, most notably biofilm formation (discussed below). The results demonstrate that the flagellar hierarchy controls the transcription of non-flagellar genes that contribute to other aspects of the $V$. cholerae lifecycle besides motility (Syed, Beyhan et al. 2009).

\section{Chemotaxis and virulence}

Chemotaxis controls flagellar rotation in response to environmental factors, and thus is intimately tied to motility. Chemoattractants stimulate the chemotaxis machinery to cause increased clockwise $(\mathrm{CW})$ rotation of the flagellum, while chemorepellants enable increased counter-clockwise (CCW) rotation (Armitage 1999; Butler and Camilli 2005). The net result of these effects on flagellar rotation is net swimming towards chemoattractants and away from chemorepellants (Falke, Bass et al. 1997; Armitage 1999). V. cholerae encodes three clusters of chemotaxis proteins (Heidelberg, Eisen et al. 2000), but the cluster that is embedded within the flagellar gene cluster (within the Class II flhA operon: cheY3, cheZ, cheA2, cheB2, and cheW1) appears to be the major chemotaxis machinery that controls flagellar rotation under most conditions (Camilli and Mekalanos 1995; Hyakutake, Homma et al. 2005). Methyl-accepting chemotaxis proteins (MCPs) in the cytoplasmic membrane interact with chemoattractant/repellants and the signal is transmitted through CheA to CheY via phosphorylation. Phospho-CheY then interacts with the C-ring of the flagellum, which causes a reversion from $\mathrm{CCW}$ to $\mathrm{CW}$ rotation, resulting in a change of swimming direction. CheB and CheW are involved in modulating the signal transduction pathway (Freter and O'Brien 1981; Alm and Manning 1990; Everiss, Hughes et al. 1994; Harkey, Everiss et al. 1994; Lee, Butler et al. 2001; Banerjee, Das et al. 2002; Hyakutake, Homma et al. 2005).

Interestingly, $V$. cholerae in stool exhibit a transient hyper-infectious phenotype predicted to facilitate epidemic spread of cholera, and transcription profiling revealed a transient repression of chemotaxis genes (specifically cheW) in these bacteria (Merrell, Butler et al. 2002). In the infant mouse model, non-chemotactic $V$. cholerae are able to outcompete chemotactic $V$. cholerae for intestinal colonization, indicating that the repression of chemotaxis in stool bacteria enhances epidemic spread (Butler and Camilli 2004; Butler, Nelson et al. 2006). Preventing phosphorylation of CheY prevents chemotactic signal transduction to the flagellum and biases it toward CCW flagellar rotation (and hence longer periods of swimming in a straight direction). The flagellum can also be biased toward CW flagellar rotation (and shorter periods of swimming in a straight direction) by the introduction of mutations into CheY that inhibit its dephosphorylation. Within the intestine, only the CCW-biased $V$. cholerae dramatically outcompete chemotactic $V$. cholerae, whereas the CW-biased bacteria are defective for intestinal colonization (Butler and Camilli 2004). Chemotactic $V$. cholerae colonize the distal end of the small intestine, whereas the CCWbiased non-chemotactic $V$. cholerae colonize the entire length of the small intestine. These results suggest that chemotaxis normally facilitates the recognition of chemoattractants within the distal small intestine or, alternatively, the recognition of chemorepellants within the proximal small intestine. 


\section{Biofilm formation}

$V$. cholerae readily forms biofilms in the laboratory, and it is generally thought that $V$. cholerae predominantly exists as biofilms associated with various surfaces in the aquatic environment, including close associations with shellfish and zooplankton (Costerton, Lewandowski et al. 1995; Watnick and Kolter 1999; Faruque, Biswas et al. 2006; Yildiz and Visick 2009). Biofilm growth on chitinous surfaces induces competence in $V$. cholerae, facilitating horizontal gene transfer and rapid evolution in the marine environment (Blokesch and Schoolnik 2007). $V$. cholerae biofilms are more resistant to environmental stresses such as antibiotics, chlorine, protozoan grazing, and bacteriophage infection (Vess, Anderson et al. 1993; Faruque, Albert et al. 1998; Watnick and Kolter 1999; Matz, McDougald et al. 2005). A significant amount of study has gone into understanding $V$. cholerae biofilm formation.

Biofilm formation requires an initial phase where the bacterium associates with a solid surface, followed by attachment, formation of microcolonies, and finally the formation of the mature three-dimensional biofilm structure with characteristic pillars and water channels (Costerton, Lewandowski et al. 1995; Watnick and Kolter 1999). Formation of the mature biofilm requires the expression of the Vibrio exopolysaccharide (VPS), which is the polysaccharide matrix that holds the structure together (Yildiz and Schoolnik 1999; Watnick, Lauriano et al. 2001; Lauriano, Ghosh et al. 2004). V. cholerae expressing the VPS results in obviously wrinkled ("rugose") colony morphology, and $V$. cholerae undergoes phase variation that leads to the rugose colony phenotype and enhanced biofilm formation ( Yildiz and Schoolnik 1999; Watnick, Lauriano et al. 2001; Lim, Beyhan et al. 2007). A number of regulatory factors are involved in VPS expression and biofilm formation, and one of the driving signals behind biofilm formation is increased expression of the signaling molecule cdi-GMP (Tischler and Camilli 2004; Beyhan, Tischler et al. 2006; Beyhan, Bilecen et al. 2007; Lim, Beyhan et al. 2007; Beyhan, Odell et al. 2008; Hickman and Harwood 2008; Syed, Beyhan et al. 2009; Yildiz and Visick 2009).

In an initial screen for $V$. cholerae mutants unable to form biofilms, Watnick and Kolter identified motility as a major contributor to biofilm formation (Watnick and Kolter 1999). These results suggested that flagellar-mediated motility was important to approach and colonize a surface, and also to facilitate microcolony formation. Subsequently, it was determined that the flagellar motor itself controls VPS expression, at least in some $V$. cholerae strains, because non-flagellated mutants switch to the rugose phenotype, and this is dependent on a functional motor, suggesting that the motor acts as a sensor to induce mature biofilm formation (Lauriano, Ghosh et al. 2004). The Vibrio Na+-driven motor functioning to sense environmental conditions and drive altered gene expression is not unprecedented; the $V$. parahaemolyticus $\mathrm{Na}+$-driven polar flagellar motor functions as a sensor to drive lateral flagellar synthesis (McCarter, Hilmen et al. 1988; Kawagishi, Imagawa et al. 1996).

In general, elevated levels of cdGMP drive $V$. cholerae toward enhanced VPS expression and down-regulate motility and virulence gene expression (Tischler and Camilli 2004; Yildiz and Visick 2009). Elevated cdGMP levels cause a decrease in Class III and IV flagellar transcription, and noticeable decreases in motility in soft agar assays(Beyhan, Tischler et al. 2006). These results suggest that activity of the Class III regulator FlrC may be responsive to elevated cdGMP levels. The effect of specific cdGMP synthases/phosphodiesterases on motility is 
complicated by the presence of multiple paralogs of both types of enzymes in $V$. cholerae (Lim, Beyhan et al. 2006; Beyhan, Odell et al. 2008). Moreover, the flagellar hierarchy also regulates the expression of cdGMP modulating enzymes (mentioned above), so the effect of cdGMP on flagellar synthesis and motility is likely extremely complex, involving a large number of counteracting enzymes that regulate and are regulated by the flagellar hierarchy.

\section{Conclusion}

The single polar flagellum of $V$. cholerae is assembled in a stepwise fashion of components that are tightly regulated by a flagellar transcriptional hierarchy. The study of some of the unique aspects of this flagellum are likely to yield further insight into the role of flagellar synthesis, motility, and chemotaxis on the virulence and environmental persistence of this important human pathogen. One of the most unique aspects is the sheath surrounding the flagellum, which is still mysterious. The presence and function of the multiple flagellins still needs to be elucidated. Regulation of the flagellar transcriptional hierarchy is still not understood, nor how this hierarchy regulates non-flagellar genes that influence virulence and biofilm formation. Clearly much remains to be illuminated in the study of the contribution of flagellar synthesis and motility to the lifecycle of $V$. cholerae.

\section{Acknowledgement}

Funded by NIH AI43486

\section{References}

Aizawa, S. I., G. E. Dean, et al. (1985). "Purification and characterization of the flagellar hook-basal body complex of Salmonella typhimurium." J Bacteriol 161(3): 836-849.

Allen, R. D. and P. Baumann (1971). "Structure and arrangement of flagella in species of the genus Beneckea and Photobacterium fischeri." J Bacteriol 107(1): 295-302.

Alm, R. A. and P. A. Manning (1990). "Characterization of the hlyB gene and its role in the production of the El Tor haemolysin of Vibrio cholerae O1." Mol Microbiol 4(3): 413-425.

Armitage, J. P. (1999). "Bacterial tactic responses." Adv Microb Physiol 41: 229-289.

Asai, Y., S. Kojima, et al. (1997). "Putative channel components for the fast-rotating sodiumdriven flagellar motor of a marine bacterium." J Bacteriol 179(16): 5104-5110.

Banerjee, R., S. Das, et al. (2002). "Involvement of in vivo induced cheY-4 gene of Vibrio cholerae in motility, early adherence to intestinal epithelial cells and regulation of virulence factors." FEBS Lett 532(1-2): 221-226.

Beyhan, S., K. Bilecen, et al. (2007). "Regulation of rugosity and biofilm formation in Vibrio cholerae: comparison of VpsT and VpsR regulons and epistasis analysis of vpsT, vpsR, and hapR." J Bacteriol 189(2): 388-402.

Beyhan, S., L. S. Odell, et al. (2008). "Identification and characterization of cyclic diguanylate signaling systems controlling rugosity in Vibrio cholerae." J Bacteriol 190(22): 73927405.

Beyhan, S., A. D. Tischler, et al. (2006). "Transcriptome and phenotypic responses of Vibrio cholerae to increased cyclic di-GMP level." J Bacteriol 188(10): 3600-3613. 
Blair, D. F. (2003). "Flagellar movement driven by proton translocation." FEBS Lett 545(1): 86-95.

Blair, D. F. and H. C. Berg (1990). "The MotA protein of E. coli is a proton-conducting component of the flagellar motor." Cell 60(3): 439-449.

Blokesch, M. and G. K. Schoolnik (2007). "Serogroup conversion of Vibrio cholerae in aquatic reservoirs." PLoS Pathog 3(6): e81.

Braun, T. F., S. Poulson, et al. (1999). "Function of proline residues of MotA in torque generation by the flagellar motor of Escherichia coli." J Bacteriol 181(11): 3542-3551.

Butler, S. M. and A. Camilli (2004). "Both chemotaxis and net motility greatly influence the infectivity of Vibrio cholerae." Proc Natl Acad Sci U S A 101(14): 5018-5023.

Butler, S. M. and A. Camilli (2005). "Going against the grain: chemotaxis and infection in Vibrio cholerae." Nat Rev Microbiol 3(8): 611-620.

Butler, S. M., E. J. Nelson, et al. (2006). "Cholera stool bacteria repress chemotaxis to increase infectivity." Mol Microbiol 60(2): 417-426.

Camilli, A. and J. J. Mekalanos (1995). "Use of recombinase gene fusions to identify Vibrio cholerae genes induced during infection." Mol Microbiol 18(4): 671-683.

Carpenter, P. B., D. W. Hanlon, et al. (1992). "flhF, a Bacillus subtilis flagellar gene that encodes a putative GTP-binding protein." Mol Microbiol 6(18): 2705-2713.

Carsiotis, M., D. L. Weinstein, et al. (1984). "Flagella of Salmonella typhimurium are a virulence factor in infected C57BL/6J mice." Infect Immun 46(3): 814-818.

Chevance, F. F. and K. T. Hughes (2008). "Coordinating assembly of a bacterial macromolecular machine." Nat Rev Microbiol 6(6): 455-465.

Correa, N. E., J. R. Barker, et al. (2004). "The Vibrio cholerae FlgM homologue is an antisigma28 factor that is secreted through the sheathed polar flagellum." J Bacteriol 186(14): 4613-4619.

Correa, N. E. and K. E. Klose (2005). "Characterization of enhancer binding by the Vibrio cholerae flagellar regulatory protein FlrC." J Bacteriol 187(9): 3158-3170.

Correa, N. E., C. M. Lauriano, et al. (2000). "Phosphorylation of the flagellar regulatory protein FlrC is necessary for Vibrio cholerae motility and enhanced colonization." Mol Microbiol 35(4): 743-755.

Correa, N. E., F. Peng, et al. (2005). "Roles of the regulatory proteins FlhF and FlhG in the Vibrio cholerae flagellar transcription hierarchy." J Bacteriol 187(18): 6324-6332.

Coster, T. S., K. P. Killeen, et al. (1995). "Safety, immunogenicity, and efficacy of live attenuated Vibrio cholerae O139 vaccine prototype." Lancet 345(8955): 949-952.

Costerton, J. W., Z. Lewandowski, et al. (1995). "Microbial biofilms." Annu Rev Microbiol 49: 711-745.

Dasgupta, N. and R. Ramphal (2001). "Interaction of the antiactivator FleN with the transcriptional activator FleQ regulates flagellar number in Pseudomonas aeruginosa." J Bacteriol 183(22): 6636-6644.

Dasgupta, N., M. C. Wolfgang, et al. (2003). "A four-tiered transcriptional regulatory circuit controls flagellar biogenesis in Pseudomonas aeruginosa." Mol Microbiol 50(3): 809824.

Dean, G. E., R. M. Macnab, et al. (1984). "Gene sequence and predicted amino acid sequence of the motA protein, a membrane-associated protein required for flagellar rotation in Escherichia coli." J Bacteriol 159(3): 991-999. 
Everiss, K. D., K. J. Hughes, et al. (1994). "The accessory colonization factor and toxincoregulated pilus gene clusters are physically linked on the Vibrio cholerae 0395 chromosome." DNA Seq 5(1): 51-55.

Falke, J. J., R. B. Bass, et al. (1997). "The two-component signaling pathway of bacterial chemotaxis: a molecular view of signal transduction by receptors, kinases, and adaptation enzymes." Annu Rev Cell Dev Biol 13: 457-512.

Faruque, S. M., M. J. Albert, et al. (1998). "Epidemiology, genetics, and ecology of toxigenic Vibrio cholerae." Microbiol Mol Biol Rev 62(4): 1301-1314.

Faruque, S. M., K. Biswas, et al. (2006). "Transmissibility of cholera: in vivo-formed biofilms and their relationship to infectivity and persistence in the environment." Proc Natl Acad Sci U S A 103(16): 6350-6355.

Francis, N. R., G. E. Sosinsky, et al. (1994). "Isolation, characterization and structure of bacterial flagellar motors containing the switch complex." J Mol Biol 235(4): 12611270.

Freter, R. and P. C. O'Brien (1981). "Role of chemotaxis in the association of motile bacteria with intestinal mucosa: fitness and virulence of nonchemotactic Vibrio cholerae mutants in infant mice." Infect Immun 34(1): 222-233.

Fuerst, J. A. and J. W. Perry (1988). "Demonstration of lipopolysaccharide on sheathed flagella of Vibrio cholerae O:1 by protein A-gold immunoelectron microscopy." J Bacteriol 170(4): 1488-1494.

Fukuoka, H., T. Yakushi, et al. (2005). "Assembly of motor proteins, PomA and PomB, in the $\mathrm{Na}$--driven stator of the flagellar motor." J Mol Biol 351(4): 707-717.

Gardel, C. L. and J. J. Mekalanos (1996). "Alterations in Vibrio cholerae motility phenotypes correlate with changes in virulence factor expression." Infect Immun 64(6): 22462255.

Green, J. C., C. Kahramanoglou, et al. (2009). "Recruitment of the earliest component of the bacterial flagellum to the old cell division pole by a membrane-associated signal recognition particle family GTP-binding protein." J Mol Biol 391(4): 679-690.

Guentzel, M. N. and L. J. Berry (1975). "Motility as a virulence factor for Vibrio cholerae." Infect Immun 11(5): 890-897.

Harkey, C. W., K. D. Everiss, et al. (1994). "The Vibrio cholerae toxin-coregulated-pilus gene tcpI encodes a homolog of methyl-accepting chemotaxis proteins." Infect Immun 62(7): 2669-2678.

Heidelberg, J. F., J. A. Eisen, et al. (2000). "DNA sequence of both chromosomes of the cholera pathogen Vibrio cholerae." Nature 406(6795): 477-483.

Hendrixson, D. R. and V. J. DiRita (2003). "Transcription of sigma54-dependent but not sigma28-dependent flagellar genes in Campylobacter jejuni is associated with formation of the flagellar secretory apparatus." Mol Microbiol 50(2): 687-702.

Hickman, J. W. and C. S. Harwood (2008). "Identification of FleQ from Pseudomonas aeruginosa as a c-di-GMP-responsive transcription factor." Mol Microbiol 69(2): 376-389.

Homma, M., S. Aizawa, et al. (1987). "Identification of the M-ring protein of the flagellar motor of Salmonella typhimurium." Proc Natl Acad Sci U S A 84(21): 7483-7487.

Homma, M., D. J. DeRosier, et al. (1990). "Flagellar hook and hook-associated proteins of Salmonella typhimurium and their relationship to other axial components of the flagellum." J Mol Biol 213(4): 819-832. 
Homma, M., K. Kutsukake, et al. (1990). "FlgB, FlgC, FlgF and FlgG. A family of structurally related proteins in the flagellar basal body of Salmonella typhimurium." J Mol Biol 211(2): 465-477.

Homma, M., K. Ohnishi, et al. (1987). "Identification of flagellar hook and basal body gene products (FlaFV, FlaFVI, FlaFVII and FlaFVIII) in Salmonella typhimurium." J Bacteriol 169(8): 3617-3624.

Hyakutake, A., M. Homma, et al. (2005). "Only one of the five CheY homologs in Vibrio cholerae directly switches flagellar rotation." J Bacteriol 187(24): 8403-8410.

Ikeda, T., S. Asakura, et al. (1985). "'Cap" on the tip of Salmonella flagella." J Mol Biol 184(4): 735-737.

Ikeda, T., M. Homma, et al. (1987). "Localization and stoichiometry of hook-associated proteins within Salmonella typhimurium flagella." J Bacteriol 169(3): 1168-1173.

Jobling, M. G. and R. K. Holmes (1997). "Characterization of hapR, a positive regulator of the Vibrio cholerae HA/protease gene hap, and its identification as a functional homologue of the Vibrio harveyi luxR gene." Mol Microbiol 26(5): 1023-1034.

Joslin, S. N. and D. R. Hendrixson (2009). "Activation of the Campylobacter jejuni FlgSR two-component system is linked to the flagellar export apparatus." J Bacteriol 191(8): 2656-2667.

Karlinsey, J. E., S. Tanaka, et al. (2000). "Completion of the hook-basal body complex of the Salmonella typhimurium flagellum is coupled to FlgM secretion and fliC transcription." Mol Microbiol 37(5): 1220-1231.

Kawagishi, I., M. Imagawa, et al. (1996). "The sodium-driven polar flagellar motor of marine Vibrio as the mechanosensor that regulates lateral flagellar expression." Mol Microbiol 20(4): 693-699.

Kennedy, M. J., E. L. Rosey, et al. (1997). "Characterization of flaA- and flaB- mutants of Serpulina hyodysenteriae: both flagellin subunits, FlaA and FlaB, are necessary for full motility and intestinal colonization." FEMS Microbiol Lett 153(1): 119-128.

Kenner, J. R., T. S. Coster, et al. (1995). "Peru-15, an improved live attenuated oral vaccine candidate for Vibrio cholerae O1." J Infect Dis 172(4): 1126-1129.

Klose, K. E. and J. J. Mekalanos (1998). "Differential regulation of multiple flagellins in Vibrio cholerae." J Bacteriol 180(2): 303-316.

Klose, K. E. and J. J. Mekalanos (1998). "Distinct roles of an alternative sigma factor during both free-swimming and colonizing phases of the Vibrio cholerae pathogenic cycle." Mol Microbiol 28(3): 501-520.

Klose, K. E., V. Novik, et al. (1998). "Identification of multiple sigma54-dependent transcriptional activators in Vibrio cholerae." J Bacteriol 180(19): 5256-5259.

Koerdt, A., A. Paulick, et al. (2009). "MotX and MotY are required for flagellar rotation in Shewanella oneidensis MR-1." J Bacteriol 191(16): 5085-5093.

Kojima, M., N. Nishioka, et al. (2011). "Conversion of mono-polar to peritrichous flagellation in Vibrio alginolyticus." Microbiol Immunol 55(2): 76-83.

Kojima, S. and D. F. Blair (2004). "The bacterial flagellar motor: structure and function of a complex molecular machine." Int Rev Cytol 233: 93-134.

Kusumoto, A., K. Kamisaka, et al. (2006). "Regulation of polar flagellar number by the flhF and flhG genes in Vibrio alginolyticus." J Biochem 139(1): 113-121. 
Kusumoto, A., N. Nishioka, et al. (2009). "Mutational analysis of the GTP-binding motif of FlhF which regulates the number and placement of the polar flagellum in Vibrio alginolyticus." J Biochem 146(5): 643-650.

Kusumoto, A., A. Shinohara, et al. (2008). "Collaboration of FlhF and FlhG to regulate polarflagella number and localization in Vibrio alginolyticus." Microbiology 154(Pt 5): 1390-1399.

Lauriano, C. M., C. Ghosh, et al. (2004). "The sodium-driven flagellar motor controls exopolysaccharide expression in Vibrio cholerae." J Bacteriol 186(15): 4864-4874.

Lee, S. H., S. M. Butler, et al. (2001). "Selection for in vivo regulators of bacterial virulence." Proc Natl Acad Sci U S A 98(12): 6889-6894.

Lim, B., S. Beyhan, et al. (2006). "Cyclic-diGMP signal transduction systems in Vibrio cholerae: modulation of rugosity and biofilm formation." Mol Microbiol 60(2): 331-348.

Lim, B., S. Beyhan, et al. (2007). "Regulation of Vibrio polysaccharide synthesis and virulence factor production by $\mathrm{CdgC}$, a GGDEF-EAL domain protein, in Vibrio cholerae." J Bacteriol 189(3): 717-729.

Lloyd, S. A., H. Tang, et al. (1996). "Torque generation in the flagellar motor of Escherichia coli: evidence of a direct role for FliG but not for FliM or FliN." J Bacteriol 178(1): 223-231.

Martinez, R. M., M. N. Dharmasena, et al. (2009). "Characterization of two outer membrane proteins, FlgO and FlgP, that influence vibrio cholerae motility." J Bacteriol 191(18): 5669-5679.

Matz, C., D. McDougald, et al. (2005). "Biofilm formation and phenotypic variation enhance predation-driven persistence of Vibrio cholerae." Proc Natl Acad Sci U S A 102(46): 16819-16824.

McCarter, L., M. Hilmen, et al. (1988). "Flagellar dynamometer controls swarmer cell differentiation of V. parahaemolyticus." Cell 54(3): 345-351.

McCarter, L. L. (1994). "MotX, the channel component of the sodium-type flagellar motor." J Bacteriol 176(19): 5988-5998.

McCarter, L. L. (1994). "MotY, a component of the sodium-type flagellar motor." J Bacteriol 176(14): 4219-4225.

Merrell, D. S., S. M. Butler, et al. (2002). "Host-induced epidemic spread of the cholera bacterium." Nature 417(6889): 642-645.

Moisi, M., C. Jenul, et al. (2009). "A novel regulatory protein involved in motility of Vibrio cholerae." J Bacteriol 191(22): 7027-7038.

Montie, T. C., D. Doyle-Huntzinger, et al. (1982). "Loss of virulence associated with absence of flagellum in an isogenic mutant of Pseudomonas aeruginosa in the burnedmouse model." Infect Immun 38(3): 1296-1298.

Morris, D. C., F. Peng, et al. (2008). "Lipidation of an FlrC-dependent protein is required for enhanced intestinal colonization by Vibrio cholerae." J Bacteriol 190(1): 231-239.

Niehus, E., H. Gressmann, et al. (2004). "Genome-wide analysis of transcriptional hierarchy and feedback regulation in the flagellar system of Helicobacter pylori." Mol Microbiol 52(4): 947-961.

Okabe, M., T. Yakushi, et al. (2001). "Cloning and characterization of motX, a Vibrio alginolyticus sodium-driven flagellar motor gene." J Biochem 130(6): 879-884. 
Okabe, M., T. Yakushi, et al. (2005). "Interactions of MotX with MotY and with the PomA/PomB sodium ion channel complex of the Vibrio alginolyticus polar flagellum." J Biol Chem 280(27): 25659-25664.

Okabe, M., T. Yakushi, et al. (2002). "MotX and MotY, specific components of the sodiumdriven flagellar motor, colocalize to the outer membrane in Vibrio alginolyticus." Mol Microbiol 46(1): 125-134.

Okunishi, I., I. Kawagishi, et al. (1996). "Cloning and characterization of motY, a gene coding for a component of the sodium-driven flagellar motor in Vibrio alginolyticus." J Bacteriol 178(8): 2409-2415.

Prouty, M. G., N. E. Correa, et al. (2001). "The novel sigma54- and sigma28-dependent flagellar gene transcription hierarchy of Vibrio cholerae." Mol Microbiol 39(6): 1595-1609.

Richardson, K. (1991). "Roles of motility and flagellar structure in pathogenicity of Vibrio cholerae: analysis of motility mutants in three animal models." Infect Immun 59(8): 2727-2736.

Rui, H., J. M. Ritchie, et al. (2010). "Reactogenicity of live-attenuated Vibrio cholerae vaccines is dependent on flagellins." Proc Natl Acad Sci U S A 107(9): 4359-4364.

Salvetti, S., E. Ghelardi, et al. (2007). "FlhF, a signal recognition particle-like GTPase, is involved in the regulation of flagellar arrangement, motility behaviour and protein secretion in Bacillus cereus." Microbiology 153(Pt 8): 2541-2552.

Sato, K. and M. Homma (2000). "Functional reconstitution of the $\mathrm{Na}(+)$-driven polar flagellar motor component of Vibrio alginolyticus." J Biol Chem 275(8): 5718-5722.

Sato, K. and M. Homma (2000). "Multimeric structure of PomA, a component of the Na+driven polar flagellar motor of vibrio alginolyticus." J Biol Chem 275(26): 20223-20228.

Schmitt, C. K., S. C. Darnell, et al. (1994). "Mutation of flgM attenuates virulence of Salmonella typhimurium, and mutation of fliA represses the attenuated phenotype." J Bacteriol 176(2): 368-377.

Schoenhals, G. J. and R. M. Macnab (1996). "Physiological and biochemical analyses of FlgH, a lipoprotein forming the outer membrane L ring of the flagellar basal body of Salmonella typhimurium." J Bacteriol 178(14): 4200-4207.

Sjoblad, R. D., C. W. Emala, et al. (1983). "Invited review: bacterial flagellar sheaths: structures in search of a function." Cell Motil 3(1): 93-103.

Stader, J., P. Matsumura, et al. (1986). "Nucleotide sequence of the Escherichia coli motB gene and site-limited incorporation of its product into the cytoplasmic membrane." J Bacteriol 166(1): 244-252.

Stolz, B. and H. C. Berg (1991). "Evidence for interactions between MotA and MotB, torquegenerating elements of the flagellar motor of Escherichia coli." J Bacteriol 173(21): 7033-7037.

Syed, K. A., S. Beyhan, et al. (2009). "The Vibrio cholerae flagellar regulatory hierarchy controls expression of virulence factors." J Bacteriol 191(21): 6555-6570.

Terashima, H., H. Fukuoka, et al. (2006). "The Vibrio motor proteins, MotX and MotY, are associated with the basal body of $\mathrm{Na}$-driven flagella and required for stator formation." Mol Microbiol 62(4): 1170-1180.

Terashima, H., M. Koike, et al. (2010). "The flagellar basal body-associated protein FlgT is essential for a novel ring structure in the sodium-driven Vibrio motor." J Bacteriol 192(21): 5609-5615. 
Terashima, H., S. Kojima, et al. (2008). "Flagellar motility in bacteria structure and function of flagellar motor." Int Rev Cell Mol Biol 270: 39-85.

Tischler, A. D. and A. Camilli (2004). "Cyclic diguanylate (c-di-GMP) regulates Vibrio cholerae biofilm formation." Mol Microbiol 53(3): 857-869.

Ueno, T., K. Oosawa, et al. (1992). "M ring, S ring and proximal rod of the flagellar basal body of Salmonella typhimurium are composed of subunits of a single protein, FliF." J Mol Biol 227(3): 672-677.

Vess, R. W., R. L. Anderson, et al. (1993). "The colonization of solid PVC surfaces and the acquisition of resistance to germicides by water micro-organisms." J Appl Bacteriol 74(2): 215-221.

Watnick, P. I. and R. Kolter (1999). "Steps in the development of a Vibrio cholerae El Tor biofilm." Mol Microbiol 34(3): 586-595.

Watnick, P. I., C. M. Lauriano, et al. (2001). "The absence of a flagellum leads to altered colony morphology, biofilm development and virulence in Vibrio cholerae O139." Mol Microbiol 39(2): 223-235.

Weinstein, D. L., M. Carsiotis, et al. (1984). "Flagella help Salmonella typhimurium survive within murine macrophages." Infect Immun 46(3): 819-825.

Yildiz, F. H. and G. K. Schoolnik (1999). "Vibrio cholerae O1 El Tor: identification of a gene cluster required for the rugose colony type, exopolysaccharide production, chlorine resistance, and biofilm formation." Proc Natl Acad Sci U S A 96(7): 4028-4033.

Yildiz, F. H. and K. L. Visick (2009). "Vibrio biofilms: so much the same yet so different." Trends Microbiol 17(3): 109-118.

Yonekura, K., S. Maki-Yonekura, et al. (2003). "Complete atomic model of the bacterial flagellar filament by electron cryomicroscopy." Nature 424(6949): 643-650.

Yoon, S. S. and J. J. Mekalanos (2008). "Decreased potency of the Vibrio cholerae sheathed flagellum to trigger host innate immunity." Infect Immun 76(3): 1282-1288.

Yorimitsu, T., Y. Asai, et al. (2000). "Intermolecular cross-linking between the periplasmic Loop3-4 regions of PomA, a component of the Na+-driven flagellar motor of Vibrio alginolyticus." J Biol Chem 275(40): 31387-31391.

Yorimitsu, T., M. Kojima, et al. (2004). "Multimeric structure of the PomA/PomB channel complex in the Na+-driven flagellar motor of Vibrio alginolyticus." J Biochem 135(1): 43-51.

Zanen, G., H. Antelmann, et al. (2004). "FlhF, the third signal recognition particle-GTPase of Bacillus subtilis, is dispensable for protein secretion." J Bacteriol 186(17): 5956-5960.

Zhou, J., S. A. Lloyd, et al. (1998). "Electrostatic interactions between rotor and stator in the bacterial flagellar motor." Proc Natl Acad Sci U S A 95(11): 6436-6441.

Zhou, J., L. L. Sharp, et al. (1998). "Function of protonatable residues in the flagellar motor of Escherichia coli: a critical role for Asp 32 of MotB." J Bacteriol 180(10): 2729-2735. 


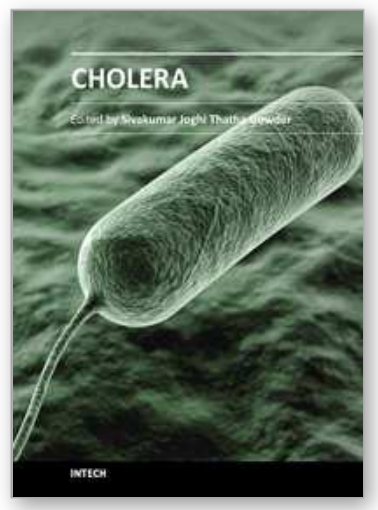

\section{Cholera}

Edited by Dr. Sivakumar Gowder

ISBN 978-953-51-0415-5

Hard cover, 218 pages

Publisher InTech

Published online 28, March, 2012

Published in print edition March, 2012

Cholera, a problem in Third World countries, is a complicated diarrheal disease caused by the bacterium Vibrio cholerae. The latest outbreak in Haiti and surrounding areas in 2010 illustrated that cholera remains a serious threat to public health and safety. With advancements in research, cholera can be prevented and effectively treated. Irrespective of "Military" or "Monetary" power, with one's "Own Power", we can defeat this disease. The book "Cholera" is a valuable resource of power (knowledge) not only for cholera researchers but for anyone interested in promoting the health of people. Experts from different parts of the world have contributed to this important work thereby generating this power. Key features include the history of cholera, geographical distribution of the disease, mode of transmission, Vibrio cholerae activities, characterization of cholera toxin, cholera antagonists and preventive measures.

\section{How to reference}

In order to correctly reference this scholarly work, feel free to copy and paste the following:

Anastasia R. Rugel and Karl E. Klose (2012). Vibrio cholerae Flagellar Synthesis and Virulence, Cholera, Dr. Sivakumar Gowder (Ed.), ISBN: 978-953-51-0415-5, InTech, Available from: http://www.intechopen.com/books/cholera/vibrio-cholerae-flagellar-synthesis-and-virulence

\section{INTECH}

open science | open minds

\section{InTech Europe}

University Campus STeP Ri

Slavka Krautzeka 83/A

51000 Rijeka, Croatia

Phone: +385 (51) 770447

Fax: +385 (51) 686166

www.intechopen.com

\section{InTech China}

Unit 405, Office Block, Hotel Equatorial Shanghai

No.65, Yan An Road (West), Shanghai, 200040, China

中国上海市延安西路65号上海国际贵都大饭店办公楼 405 单元

Phone: $+86-21-62489820$

Fax: +86-21-62489821 
(C) 2012 The Author(s). Licensee IntechOpen. This is an open access article distributed under the terms of the Creative Commons Attribution 3.0 License, which permits unrestricted use, distribution, and reproduction in any medium, provided the original work is properly cited. 